\title{
Tipo de derivación urinaria en el paciente llevado a cistectomía radical, participación del urólogo y tasa de filtración de la anastomosis intestinal
}

\section{Type of urinary diversion in the patient led to a radical cystectomy, participation of the urologist, and filtration rate of intestinal anastomosis}

Juan Luis Jaramillo Valencia ${ }^{1}$ Andrés Felipe Chica González ${ }^{2}$ Rafael Ignacio Castellanos Acosta ${ }^{3}$

\footnotetext{
${ }^{1}$ Residente Urología IV año, Universidad CES, Medellín, Colombia

2 Residente Urología III año, Universidad CES, Medellín, Colombia

${ }^{3}$ Urólogo, Jefe Postgrado Urología, Universidad CES,

Medellín, Colombia
}

Address for correspondence Andrés Felipe Chica González, Residente Urología III año, Universidad CES, Medellín, Colombia (e-mail: andreschicauro@gmail.com).

Urol Colomb 2018;27:63-66.

\section{Resumen}

Palabras clave

- cáncer vesical invasivo

- cistectomía

- vejiga urinaria

- filtración

- derivación urinaria

- intestino delgado
Objetivo Conocer la prevalencia de cistectomías radicales que se realizan en centros especializados en Colombia, definiendo tipo de derivación intestinal, participantes en su creación, segmento intestinal utilizado y tasa de filtración.

Materiales y métodos Se realizó una encuesta a instituciones de salud colombianas que realizan cistectomías radicales de manera rutinaria, se analizaron variables como número de procedimientos por año, segmento intestinal utilizado, tipo de especialidad participante en la anastomosis intestinal y la tasa de filtración de esta.

Resultados Quince instituciones colombianas respondieron la encuesta, el número de cistectomías realizadas por año fue: 5/15 (33,3\%) más de 15 cirugías al año, 4/15 (26,6\%) entre 11 y 15 procedimientos al año, 3/15 (20\%) entre 5 y 10 y otro $3 / 15(20 \%)$ entre 1 y 5 cistectomías al año. El 93,3\% de las instituciones realizan Bricker como derivación más común; solo una institución (6,7\%) lleva a cabo ureterostomías cutáneas. Con respecto a los participantes en la creación de la anastomosis de las 14 instituciones, en $9(64,2 \%)$ es realizada por cirujano general, en $4(28,5 \%)$ la lleva a cabo un urólogo y en una $(7,4 \%)$ la derivación es realizada por coloproctólogo. La gran mayoría de los centros tiene una incidencia baja de filtración intestinal.

Conclusiones En la gran mayoría de las instituciones colombianas el cirujano general y en menor medida el urólogo participan en la creación de la anastomosis intestinal como parte del protocolo de la institución. La filtración es una complicación poco frecuente pero con alta morbimortalidad. Se requiere de entrenamiento por parte del urólogo en formación para lograr mejores resultados. received November 26, 2016 accepted April 16, 2017 published online January 10, 2018
DOI https://doi.org/ 10.1016/j.uroco.2017.04.003. ISSN 0120-789X. eISSN 2027-0119.
Copyright ( 2018, Sociedad Colombiana License terms de Urología. Publicado por Thieme Revinter Publicações Ltda., Rio de Janeiro, Brazil. Todos los derechos reservados.

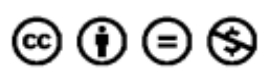




\begin{abstract}
Keywords

- invasive bladder cancer

- cystectomy

- urinary bladder

- filtration

- urinary diversion

- small intestine

Objective To determine the prevalence of radical cystectomies that are performed in specialist centres in Colombia, defining the type of intestinal derivation, participants in its creation, intestinal segment used, and filtration rate.

Materials and methods A survey was conducted in Colombian health institutions that routinely perform radical cystectomies. An analysis was made of the variables, such as number of procedures per year, intestinal segment used, specialty participating in the intestinal anastomosis, and the rate of filtration.

Results A total of 15 health institutions responded to the survey. The number of cystectomies performed per year was; $5 / 15$ (33.3\%) with greater than 15 surgeries per year, $4 / 15$ (26.6\%) with between 11 and 15 procedures per year, 3/15 (20\%) between 5-10/ year, and another $3 / 15(20 \%)$ between $1-5$ cystectomies per year. Most $(93.3 \%)$ of the institutions performed a Bricker as the most common derivation, with only one institution (6.7\%) performing cutaneous ureterostomies. As regards the participants in the creation of the anastomosis of the 14 institutions, $9(64.2 \%)$ were performed by a general surgeon, by a urologist in 4 (28.5\%), and a coloproctologist performed the shunt in one (7.4\%). The vast majority of centres have a low incidence of intestinal filtration.

Conclusions In the great majority of Colombian institutions, the general surgeon, and to a lesser extent the urologist, participate in the creation of intestinal anastomosis as part of the protocol of the institution. Filtration is a rare complication, but with a high morbidity and mortality. Training by the urologist during training is required to achieve better results.
\end{abstract}

El cáncer de vejiga es la neoplasia maligna más frecuente del tracto urinario, a nivel mundial es la novena más diagnosticada con 430.000 casos nuevos al año en todo el mundo. ${ }^{1}$ Cuando hay compromiso de la capa muscular la cistectomía radical con derivación urinaria es el tratamiento estándar para este estadio de la enfermedad. ${ }^{2,3}$ Este es un procedimiento invasivo que no está libre de complicaciones, con alta mortalidad y morbilidad postoperatorias. ${ }^{4,5}$ La frecuencia de las complicaciones intra- $y$ postoperatorias está relacionada con la experiencia del cirujano y las variables clínicas de cada paciente. En grandes series se describen tasas de complicaciones hasta del $41 \%$, y tasas de mortalidad perioperatoria entre el 0,7 y $11 \%$.

La filtración de la anastomosis intestinal posterior a la realización de la cistectomía radical con derivación urinaria puede ser devastadora debido a que se asocia a una elevada mortalidad y morbilidad. Actualmente debido al avance en los métodos de nutrición enteral, en los cuidados en las unidades de terapia intensiva, la mortalidad ha disminuido con respecto a datos observados en la década de los 60 cuando el $43 \%$ de los pacientes murieron por acontecimientos secundarios a esta entidad. Pese a los avances, este proceso patológico genera en los pacientes alteraciones en el estado de ánimo y una imagen negativa de sí mismo, lo que puede ser explicado por el tiempo prolongado que requieren estar hospitalizados, generando además aumentos en el costo del sistema de salud. ${ }^{6}$

El objetivo de nuestro trabajo es conocer la prevalencia de cistectomías radicales que se realizan en centros especializados en Colombia, definiendo el tipo de derivación intestinal, los participantes en su creación, el segmento intestinal utilizado y la tasa de filtración de la misma.

\section{Materiales y métodos}

Se diseñó una encuesta con un total de 8 preguntas, la cual se entrego a urólogos que trabajaran en centros que realizan cistectomías radicales de manera rutinaria, se analizaron variables como número de procedimientos por año, segmento intestinal utilizado, derivación urinaria más frecuente, tipo de especialidad participante en la anastomosis intestinal, tasa de filtración de esta y tipo de sutura intestinal utilizada. Además se analizó qué tanta participación tienen otras especialidades durante el procedimiento. En total fueron diligenciadas 15 encuestas de diferentes centros del país.

Los datos fueron analizados por medio de tablas dinámicas con uso de Microsoft Excel y SPSS.

\section{Resultados}

De los 15 centros a nivel nacional que respondieron a la encuesta el número de cistectomías realizadas por año fue 5/15 (33.3\%) más de 15/año, 4/15(26.6\%) entre 11 y 15 procedimientos al año, 3/15 (20\%) entre 6-10 y otro $3 / 15$ (20\%) entre 1-5 cistectomías en el año (- Tabla 1). El 93,3\% de encuestados realizan Bricker como derivación urinaria más común, solo una institución (6,7\%) lleva a cabo ureterostomías cutáneas (-Tabla $\mathbf{1}$ ).

Con respecto a los participantes en la creación de la anastomosis intestinal, de las 14 instituciones que realizaban este procedimiento, en 9 (64,2\%) de ellas la realiza el cirujano general, en 4 (28,5\%) únicamente por el urólogo y en 1 (7.4\%) la derivación es realizada por cirujano de vías digestivas y/o 
Tabla 1 Número de cistectomías realizadas por año en las instituciones encuestadas

\begin{tabular}{|l|l|l|}
\hline & $\begin{array}{l}\text { Número de } \\
\text { instituciones }\end{array}$ & $\begin{array}{l}\text { Porcentaje } \\
(\%)\end{array}$ \\
\hline $0-5$ & 3 & 20 \\
\hline $6-10$ & 3 & 20 \\
\hline $11-15$ & 4 & 26 \\
\hline Más de 15 & 5 & 33 \\
\hline
\end{tabular}

coloproctólogo. En todos los casos en los cuales el urólogo no participa en la creación de la derivación urinaria, se debe a un protocolo definido por cada institución para manipulación intestinal, el cual dicta que siempre debe participar el cirujano general en la realización de la anastomosis intestinal; en la única institución donde no está institucionalizado este protocolo, el urólogo dejó de hacer la anastomosis debido a las complicaciones postoperatorias que presentó (-Tabla 2 ).

El 92,8\% (13/14) de los centros tiene una incidencia entre el 0-5\% de tasa de filtración de la anastomosis del intestino, la institución restante reporta una prevalencia entre el 6-10\% de filtración 5 (-Fig. 1). El 100\% de los participantes utilizan íleon terminal y realizan en la mayoría de los casos derivación tipo Bricker. En 9/14 instituciones se usa de manera rutinaria sutura mecánica para realizar la anastomosis intestinal.

\section{Discusión}

Dentro de los procedimientos urológicos, la cistectomía puede ser el de mayor exigencia y uno de los que mayor habilidad quirúrgica requiere por parte del urólogo, porque no solo se está manipulando la vía urinaria, sino que se requiere de cierto entrenamiento en manejo intestinal para tomar un segmento y poder realizar una derivación, y posteriormente realizar las anastomosis intestinal y urinaria respectivas, considerando siempre evitar las complicaciones postoperatorias las cuales tienen altas tasas de morbimortalidad. Dentro de las complicaciones una que causa especial preocupación es la filtración de la anastomosis intestinal cuya presentación clásica es fiebre, signos de infección de la herida quirúrgica y elevación de los reactantes de fase aguda que se caracteriza porque se aumentan entre el quinto y el séptimo día del postoperatorio. En algunos casos los pacientes se encuentran muy enfermos e incluso algunos pueden tener signos de sepsis como hipotensión, taquicardia o falla orgánica multisistémica. ${ }^{2,7}$

Tabla 2 Participación de las especialidades en la elaboración de la derivación urinaria

\begin{tabular}{|l|l|}
\hline $\begin{array}{l}\text { Participantes en } \\
\text { la anastomosis }\end{array}$ & $\begin{array}{l}\text { Número de } \\
\text { instituciones (\%) }\end{array}$ \\
\hline Cirujano general & $9(62,2)$ \\
\hline Urólogo & $4(28,5)$ \\
\hline Cirujano de vías digestivas & $1(7,4)$ \\
\hline
\end{tabular}

\section{Porcentaje de filtración de la anastomosis}

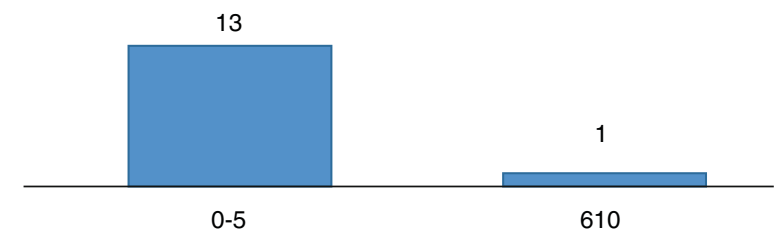

Fig. 1 Filtración de la anastomosis intestinal.

La realización de una tomografía de abdomen contrastada idealmente con la administración de contraste oral en la que se observe extravasación del medio de contraste en el sitio de la anastomosis confirma el diagnóstico, además se puede observar algunas colecciones alrededor de la anastomosis término-terminal.

Existen 2 opciones de manejo en los pacientes con filtración de la anastomosis intestinal. Una de ellas es el manejo conservador y la otra es un manejo quirúrgico por medio de laparotomía exploratoria urgente. La opción de manejo conservador se puede considerar cuando hay ausencia de peritonitis o sepsis, ${ }^{8}$ si el paciente tiene una fístula controlada, no tiene múltiples abscesos intraabdominales que sean refractarios al manejo con drenaje percutáneo y que permita adicional a este manejo intervencionista brindar al paciente manejo con antibiótico dirigido según el microorganismo del que se obtenga crecimiento en el cultivo. ${ }^{9}$ En caso de que se presente infección de la herida quirúrgica lo que se debe hacer son curaciones periódicas y dejar que esta cierre por segunda intención.

Todos los pacientes se deben dejar sin vía oral, se debe colocar una sonda nasogástrica o gastrostomía en algunos casos, con el objetivo de lograr una descompresión proximal; en caso de que desarrolle un fístula intestinal la nutrición parenteral total aumenta la tasa de cierre espontáneo al ocasionar una hipoactividad del intestino.

Dentro de las opciones adicionales en el manejo conservador de las fistulas existen datos sobre el uso de análogos de la somatostatina. Este tipo de medicamentos análogos han demostrado que disminuyen el débito de la fístula por lo que es fácil el manejo de los trastornos de líquidos, electrólitos y proteínas; la ventaja terapéutica con respecto a la reducción del tiempo de cierre de la fístula no se ha demostrado de forma consistente en los ensayos clínicos. ${ }^{10,11}$ Cuando son tratados de forma conservadora, solo el $50 \%$ de las fístulas intestinales postoperatorias cierran espontáneamente dentro de las 4-6 semanas en ausencia de obstrucción distal o la pérdida de la continuidad intestinal, el resto de los paciente si no desarrollan sepsis suelen tener una respuesta adecuada a la cirugía electiva de corrección de la fístula intestinal. ${ }^{8}$

La decisión de una laparotomía urgente aguda se justifica en pacientes que presenten una peritonitis o signos de sepsis con abscesos intraperitoneales que no han podido o no son susceptibles de drenaje percutáneo. ${ }^{8}$ El objetivo de la laparotomía es limpiar la cavidad abdominal y la pelvis de cualquier absceso loculado utilizando abundante cantidad de 
líquidos, con el objetivo de controlar la fuente de contaminación que por lo general es a través de la creación de una colostomía proximal.

El desarrollo de una fístula en los 2 primeros días es generalmente el resultado de un error técnico y si se identifica de manera oportuna se puede realizar una reanastomosis intestinal. En nuestro estudio, la gran mayoría de las instituciones tiene protocolizada la participación del cirujano general o subespecialista en vías digestivas para la creación de la derivación y realización de la anastomosis término-terminal; esto puede ser explicado por la falta de entrenamiento de los urólogos en formación en la manipulación intestinal y lo grave de las complicaciones en caso de que se presente filtración de la anastomosis. Además se observó que la gran mayoría de las instituciones utiliza grapadora mecánica para realizar la sutura, explicado por la disminución del tiempo operatorio mediante esta técnica.

La técnica quirúrgica al realizar la anastomosis intestinal es crucial para evitar el desarrollo de una fístula enterocutánea. Una exposición adecuada de los segmentos que se van a anastomosar, el mantenimiento de un excelente suministro de sangre a los extremos seccionados y el no realizar suturas con presión excesiva sobre los tejidos que puedan ocasionar estrangulación permiten que la anastomosis intestinal tenga mayor probabilidad de funcionar de manera adecuada. ${ }^{6}$

\section{Conclusiones}

En la gran mayoría de las instituciones colombianas el cirujano general y en menor medida el urólogo participan en la creación de la anastomosis intestinal para cistectomía radical con derivación urinaria como parte del protocolo de la institución. La filtración intestinal es una complicación asociada al procedimiento, que aunque es poco frecuente, es una entidad con alta morbimortalidad. Se requiere de entrenamiento por parte del urólogo en formación en manipulación intestinal, técnica quirúrgica y manejo de complicaciones para lograr mejores resultados en procedimientos urológicos que impliquen utilizar el intestino y de esta manera llegar a realizar estos procedimientos con mayor autonomía.

\section{Responsabilidades éticas}

\section{Protección de personas y animales}

Los autores declaran que para esta investigación no se han realizado experimentos en seres humanos ni en animales.

\section{Confidencialidad de los datos}

Los autores declaran que en este artículo no aparecen datos de pacientes.

\section{Derecho a la privacidad y consentimiento informado} Los autores declaran que en este artículo no aparecen datos de pacientes.

\author{
Financiación \\ No hubo financiación. \\ Conflicto de intereses \\ No hay conflicto de intereses.
}

\section{Bibliografía}

1 Ploeg M, Aben KK.H, Kiemeney LA. The present and future burden of urinary bladder cancer in the world. World J Urol 27;2009: 289-293

2 Niegisch G, Albers P, Rabenalt R. Perioperative complications and oncological safety of robot-assisted (RARC) vs. open radical cystectomy (ORC). Urol Oncol 32;2014:966-974

3 Stenzl A, Cowan NC, de Santis M, Kuczyk MA, Merseburger AS, Ribal MJ, et al. Treatment of muscle-invasive and metastatic bladder cancer: Update of the EAU guidelines. Eur Urol 59;2011:1009-1018

4 De Nunzio C, Cindolo L, Leonardo C, Antonelli A, Ceruti C, Franco G, et al. Analysis of radical cystectomy and urinary diversion complications with the Clavien classification system in an Italian real life cohort. Eur J Surg Oncol 39;2013:792-798

5 Takada N, Abe T, Shinohara N, Sazawa A, Maruyama S, Shinno Y, et al. Peri-operative morbidity and mortality related to radical cystectomy: A multi- institutional retrospective study in Japan. BJU Int 110 (11Pt B): 2012:E756-E764

6 Falconi M, Pederzoli P. The relevance of gastrointestinal fistulae in clinical practice: A review. Gut 49(Suppl 42001:iv2-iv10

7 Harraz AM, Osman Y, El-Halwagy S, Laymon M, Mosbah A, AbolEnein $\mathrm{H}$, et al. Risk factors of hospital readmission after radical cystectomy and urinary diversion: Analysis of a large contemporary series. BJU Int 115;2015:94-100

8 Schein M. Postoperative small bowel leak. Br J Surg 86;1999: 979-980

9 Schein M, Decker GA. Postoperative external alimentary tract fistulas. Am J Surg 161;1991:435-438

10 Jamil M, Ahmed U, Sobia H. Role of somatostatin analogues in the management of enterocutaneous fistulae. J Coll Physicians Surg Pak 14;2004:237-240

11 Martineau P, Shwed JA, Denis R. Is octreotide a new hope for enterocutaneous and external pancreatic fistulas closure? Am J Surg 172;1996:386-395 